\title{
EM-Based High Speed Wireless Sensor Networks for Underwater Surveillance and Target Tracking
}

\author{
Kumudu Munasinghe, ${ }^{1}$ Mohammed Aseeri, ${ }^{2}$ Sultan Almorqi, ${ }^{2}$ Md. Farhad Hossain, ${ }^{3}$ \\ Musbiha Binte Wali, ${ }^{3}$ and Abbas Jamalipour ${ }^{4}$ \\ ${ }^{1}$ Faculty of Education, Science, Technology and Mathematics, University of Canberra, Canberra, ACT 261, Australia \\ ${ }^{2}$ National Centre for Sensors and Defense Systems Technologies, King Abdulaziz City of Science and Technology, Riyadh, Saudi Arabia \\ ${ }^{3}$ Department of Electrical and Electronic Engineering, Bangladesh University of Engineering and Technology, Dhaka 1000, Bangladesh \\ ${ }^{4}$ School of Electrical and Information Engineering, University of Sydney, Sydney, NSW 2006, Australia
}

Correspondence should be addressed to Kumudu Munasinghe; kumudu.munasinghe@canberra.edu.au

Received 17 June 2016; Revised 16 November 2016; Accepted 14 December 2016; Published 26 February 2017

Academic Editor: José A. Somolinos

Copyright (C) 2017 Kumudu Munasinghe et al. This is an open access article distributed under the Creative Commons Attribution License, which permits unrestricted use, distribution, and reproduction in any medium, provided the original work is properly cited.

\begin{abstract}
Underwater Wireless Sensor Networks (UWSNs) are considered as tangible, low cost solution for underwater surveillance and exploration. Existing acoustic wave-based UWSN systems fail to meet the growing demand for fast data rates required in military operations, oil/gas exploration, and oceanographic data collection. Electromagnetic (EM) wave-based communication systems, on the other hand, have great potential for providing high speed data rates in such scenarios. This paper will (1) discuss the challenges faced in the utilization of EM waves for the design of tactical underwater surveillance systems and (2) evaluate several EM wave-based three-dimensional (3D) UWSN architectures differing in topologies and/or operation principles on the performance of localization and target tracking. To the best of our knowledge, this is the first of its kind in the field of underwater communications where underwater surveillance techniques for EM wave-based high speed UWSNs have been investigated. Thus, this will be a major step towards achieving future high speed UWSNs.
\end{abstract}

\section{Introduction}

In terms of underwater surveillance, Underwater Wireless Sensor Networks (UWSNs) are considered as a tangible, low cost solution $[1,2]$. In these networks, sensor nodes are deployed at various depths in underwater and communicate with other networked floating nodes (e.g., buoys) on the surface and other communications equipment installed in maritime and airborne vehicles (e.g., ships, aircraft, and satellites) $[3,4]$. Most of today's underwater surveillance systems are equipped with sonar-array based target tracking algorithms [5-8]. Sonar arrays are based on acoustic wave technology since they are capable of providing long-range communications in underwater $[9,10]$. Acoustic waves however result in poor performance in shallow water environments and have extremely low data rates [11] and therefore deemed impractical for on-demand real-time target tracking applications. Moreover, acoustic transmission is affected by multipath propagation, susceptibility to environmental noise, turbidity, salinity gradients, pressure gradients, and adverse impact on marine life. Therefore, electromagnetic (EM) transmissions have been considered as a better alternative for UWSNs [12]. Despite having a relatively shorter range, EM technology is a promising technology for UWSNs as they have the ability to provide much higher data rates than those achievable with acoustic waves in harsh environments with no direct path. This new breed of UWSNs can provide real-time deep-sea oil and gas explorations, military surveillance, search and rescue operations, and environmental monitoring. A comparison on the advantages and disadvantages of acoustic and EM wavebased communications is presented in Table 1.

By and large, underwater surveillance systems used within a military context have three main characteristics. The three key characteristics are detection, identification, and tracking submerged targets (localization). Target detection is how the network identifies a potential target within its 
TABLE 1: Advantages, disadvantages, and challenges of underwater networking using acoustic and EM waves [12-14].

\begin{tabular}{|c|c|c|}
\hline Particulars & Acoustic wave & EM wave \\
\hline Advantages & $\begin{array}{l}\text { (i) Significantly lower signal attenuation } \\
\text { (ii) Longer transmission area in the range } \\
\text { of } \mathrm{km} \\
\text { (iii) Can function in the absence of } \\
\text { line-of-sight (LOS) path between } \\
\text { transmitting and receiving nodes }\end{array}$ & $\begin{array}{c}\text { (i) Large bandwidth } \\
\text { Mbps } \\
\text { (ii) High data rates in the range of few } \\
\text { (iii) Faster response due to higher } \\
\text { propagation speed and significantly lower } \\
\text { delay } \\
\text { (iv) LOS for communication is not } \\
\text { essential } \\
\text { (v) No need of clear water } \\
\text { (vi) No noticeable impact of underwater } \\
\text { environment, such as temperature, } \\
\text { turbidity, salinity, bubbles, and pressure } \\
\text { gradients and thus improving robustness } \\
\text { in unpredictable underwater } \\
\text { environment } \\
\text { (vii) Not affected by sediments and } \\
\text { aeration } \\
\text { (viii) Immune to other noise except } \\
\text { electromagnetic interference (EMI) } \\
\text { (ix) Lower Doppler shift } \\
\text { (x) More reliable communication } \\
\text { (xi) Can cross water-to-air or } \\
\text { water-to-earth boundaries easily } \\
\text { (xii) No impact on marine life } \\
\text { (xiii) Lower cost of nodes } \\
\text { (xiv) Good performance in shallow water } \\
\text { (xv) Higher attenuation is beneficial in an } \\
\text { environment of multiuser interference }\end{array}$ \\
\hline
\end{tabular}

(i) Significantly slower response as propagation speed is much lower $(1500 \mathrm{~m} / \mathrm{s})$ than that of EM wave

(ii) Significantly lower data rate (up to $20 \mathrm{kbps}$ ) as bandwidth is low

(iii) Surface repeater is required as strong reflections and attenuation occurs in crossing water/air boundary

Disadvantages

Challenges (iv) Poor performance in shallow water

(v) Less reliable and robust communication as easily affected by turbidity, ambient noise, temperature, salinity, and pressure gradients

(vi) Adverse impact on the marine life and ecosystem

(vii) Higher cost of network nodes

(i) Higher and variable latency

(ii) Difficult time synchronization due to variable delay

(iii) Higher bit error rate

(iv) Multipath propagation and fading

(v) Easy signal corruption due to ambient noise (i) Easy to be affected by EMI

(ii) Higher attenuation, which increases with the salinity of water

(iii) Limited communication range in high data rate applications (e.g., $50 \mathrm{~m}$ for $150 \mathrm{kbps}$ and less than $10 \mathrm{~m}$ for Mbps range)

(iv) Dense deployment of nodes is required for higher frequency range

(vi) Mobility of nodes

vicinity. Target identification is the classification of the aforementioned target (i.e., submarines, divers, naval mines, sea animals, etc.). Once a potential target is identified, localization techniques are required for tracking of its movements. UWSN localization is a very challenging problem due to the unavailability of the Global Positioning System (GPS) underwater. Energy efficiency and access delay are two major constraints of any UWSN [13-15]. On the other hand, high throughput with low packet collisions is desirable for modern applications [12]. Fair resource allocation among multiple 


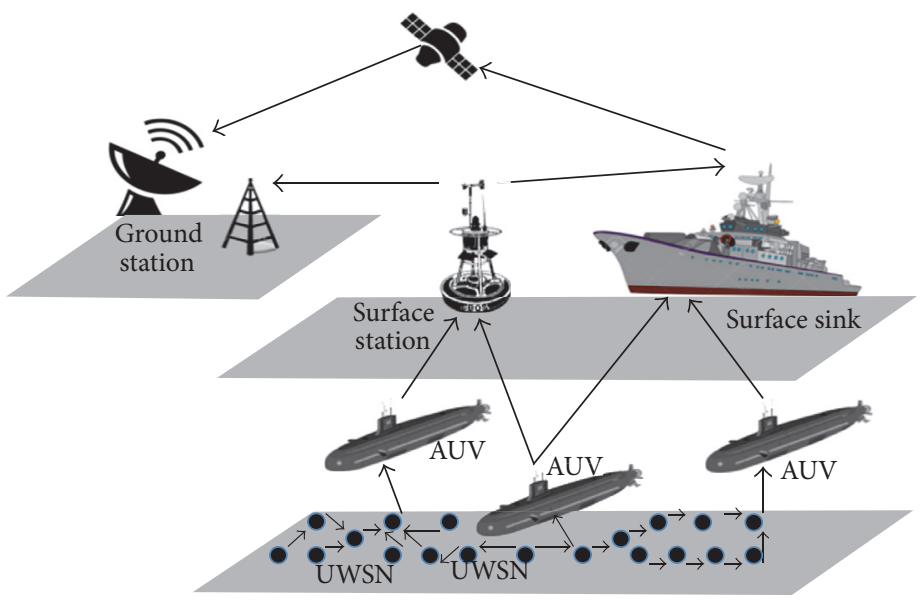

FIGURE 1: FORCEnet project from the US Navy's unmanned undersea vehicles (UUV) program office.

nodes is another critical factor for effective operation of UWSNs [10]. In addition, the sensor node topology (node distribution) and network architecture play a key role in the design process of these surveillance techniques [13]. For example, node distribution relates to how the sensor nodes are distributed with respect to the characteristics of a particular target, which also needs attention during the UWSN design process.

Our motivation is driven by the fact that existing surveillance systems designed for terrestrial WSNs are inappropriate for EM-based UWSNs due to the fundamental differences between characteristics of the two mediums. Furthermore, due to the marked differences between the propagation characteristics of acoustic and EM waves, most existing underwater surveillance systems that are based on acoustic technology will not be applicable for EM wave-based communications. It is therefore extremely rare to find existing works on underwater surveillance systems for EM wave-based UWSNs. Thus, it is of utmost significance to divert considerable research effort in developing underwater surveillance systems suitable for EM wave-based high speed UWNSs, which is the foremost objective of this paper. This paper investigates (1) the opportunities and challenges of using EM wave-based UWSNs supporting high speed data transmissions with a particular focus on the design of suitable tactical underwater surveillance systems and (2) evaluates several EM wave-based threedimensional (3D) UWSN architectures on the performance of localization and target tracking. To the best of our knowledge, development of appropriate underwater surveillance techniques for EM wave-based high speed UWSNs will be the first of its kind in the field of underwater communications.

The remainder of this paper is organized as follows. Next we present a literature review that discussed the challenges encountered in the design of underwater surveillance system with particular emphasis to UWSNs. Followed by this we present the system design considerations that need attention for such a design. Next we introduce the EM wave-based three-dimensional UWSN architectures for localization and target tracking followed by a discussion and conclusion.

\section{Underwater Surveillance Systems and Challenges}

Approximately $70 \%$ of the surface of the earth is covered by water. Further $97 \%$ of the aforementioned is seawater [16]. Due to the lack of efficient underwater information collecting networks, this vast area of underwater world, which is in abundance of extremely rich natural resources, has hardly been explored. Furthermore, military and political tensions between nations also call for efficient underwater surveillance systems for maritime boundary protection [16]. Issues to be considered with surveillance systems are target detection, localization, classification, and tracking. Traditionally, sonararray based systems were used for underwater target tracking. As a result, a number of sonar-array systems have been designed for this purpose [9, 16-18]. When sonar-array equipment is submerged and dragged by some sort of vessels (e.g., ship and submarine), they become unsuitable for ondemand tracking missions $[19,20]$. Further, if the platform, which it tows, breaks down, the entire system fails $[21,22]$.

2.1. WSNs for Underwater Surveillance. In order to avoid the issues of sonar-array based target tracking mechanisms, UWSNs have been proposed as an alternative solution. In terms of underwater target tracking, UWSNs seem to offer a promising approach. Low cost, rapid deployment, selforganization, and fault tolerance are the main advantages of UWSNs [23]. As a result, there has been growing interest in research and development in using UWSN as a tangible, low cost solution [13]. UWSNs typically include a large number of intercommunicating underwater devices such as sensors, buoys, gateways, sinks, anchors, and autonomous underwater vehicles (AUVs), which are coordinated for carrying out certain tasks in a collaborative manner. Such networks are often integrated with water surface sinks and stations, submarines, satellite networks, aviation systems, and onshore base stations (sinks) enabling extended functionalities [15, 16]. FORCEnet project of US Navy, as illustrated in Figure 1, is one example of such systems, where an artist's conception of their system can be found in [2]. 
2.2. Acoustic UWSN Based Surveillance Techniques. Some effort has been made for acoustic wave-based UWSN based underwater target tracking. For example, location estimation is discussed in [24]. To detect underwater target size, a maximum likelihood estimation algorithm is proposed in [25] but, however, lacks a tracking mechanism. In [26], two tracking schemes based on the distributed particle filter have been proposed for cluster based UWSNs.

The biggest downside of these tracking schemes is that they only considered two dimensions. This imposes severe limitations for applications. In response to the above, a 3D target tracking scheme with the combination of interacting multiple models is designed to solve the nonlinear and manoeuvring problems [27]. However, this scheme does not consider the energy consumption problem, which is also a limitation for practical applications. In response, Isik and Akan designed a 3D target tracking solution [28]. The arrival time of these echo messages coming from the target is used for determining the distance from the sensor to the target. Then trilateration is utilized to calculate target's position. Node's position and the above velocity will then be used for tracking the underwater subject. In [29], the waking-up sleep mechanism is utilized to save energy consumption. Despite the aforementioned advances, all of these are based on acoustic UWSNs. Current underwater acoustic communications technologies suffer from serious challenges such as susceptibility to environmental noise and require expensive signal processing to deal with the multipath acoustic channel [30].

2.3. Motivations for EM-Based UWSN Surveillance Techniques. As discussed in Table 1, due to the behaviour of acoustic waves under water, it can be justified that acoustic UWSNs are inappropriate for most modern day underwater applications. Further, an extensive survey conducted by the authors has indicated that there is no complete published works on underwater surveillance systems specifically designed for EM-based UWSNs. Due to aforementioned drawbacks of current underwater acoustic communication technologies, very recent works have proposed EM-based UWSNs as a cost-effective and reliable way forward [12].

To further justify, the advantages of EM waves over acoustic waves for UWSNs could be stated as follows. Firstly, the relatively higher channel bandwidth and data rates (up to $100 \mathrm{Mbps}$ ) of EM UWSNs are a clear benefit over relatively lower bandwidth and data rates of acoustic UWSNs (up to $20 \mathrm{kbps}$ ). Secondly, the relatively higher propagation speeds would give the EM UWSNs the capabilities such as fast detection, instantaneous tracking, and quick countermeasuring. Thirdly, unlike acoustic UWSNs, EM UWSNs are unaffected by temperature, salinity, turbidity, pressure gradients, and wind speed of the sea. Further, EM UWSNs are highly susceptible to various sources of acoustic noise (e.g., marine life at the seabed and wind speed). In addition, EM UWSNs outperform acoustic UWSNs with its capabilities of nonline of sight operation (e.g., unaffected by aeration and sediments at the seabed). Moreover, EM wave suffers less attenuation in shallow water enabling longer range UWSNs, whereas the seeming drawback of higher attenuation of EM wave in deep water can be exploited in a beneficial way for multiuser parallel data transmission enabling localized communications in UWSNs. Further, the relatively lower cost of RF nodes will further add to the aforementioned reliability making EM UWSNs a clear winner. Lastly, EM UWSNs have no known impact on the marine life and ecosystem.

Nevertheless, the biggest challenge of using EM-based radios underwater is its limited communication range due to high attenuation in water. Therefore, a relatively short communication range and a relatively large number of nodes are required to provide connectivity across large areas. Consequently, optimal node placement algorithms have to be developed. Nevertheless, since the cost of EM-based radios is significantly lower than that of acoustic-based radios, the cost of high density EM-based WSNS should not be an issue. On the other hand, because of the fundamental differences between propagation characteristics of EM wave on terrestrial and underwater channels as well as the topological differences of wireless sensor networks, it is apparent that the surveillance protocols designed for terrestrial WSNs will not operate effectively in underwater environment. Similarly, the absolute differences in generation, propagation, and detection of acoustic and EM waves clearly rule out the appropriateness of the existing acoustic surveillance techniques from being used in EM UWSNs. Some of the challenges, other than those inherent properties, which cannot be compensated by developing sophisticated schemes, of underwater communications for both acoustic and EM waves are also summarized in Table 1.

\section{System Design Considerations}

According to our comprehensive literature survey, we have found out considerable research challenges such as EM wave propagation behaviour in underwater environments, underwater channel models, physical and chemical properties of the underwater environment, water dynamics, geological distribution of seabed, and other factors influencing the UWSN performance [12-14]. Knowledge on these underwater propagation characteristics of EM waves can be directly used in the development process. Therefore, the new challenges identified in this paper are novel surveillance techniques for underwater EM-based UWSNs and the formulation of analytical models as well as development of simulation platforms for evaluating the performance of the proposed techniques. Other challenges identified are theoretical analysis to investigate the performance of the proposed surveillance technique and algorithms. Simulation tools including MATLAB, NS2, and OPNET can be used to verify the theoretical analysis results. Aqua-Sim, an NS2 based network simulator specifically designed for UWSNs by the University of Connecticut, can also be used for performance evaluation [31].

3.1. Node Topology Design Considerations. Node topology has a great effect on the performance of any acoustic UWSNs $[32,33]$. Thus, it can be inferred that underwater node/target localization as well as target tracking performance with EM communication will also be highly dependent on the node topology. Firstly, the design objectives of UWSN node topologies for mobile UWSNs would greatly differ between 


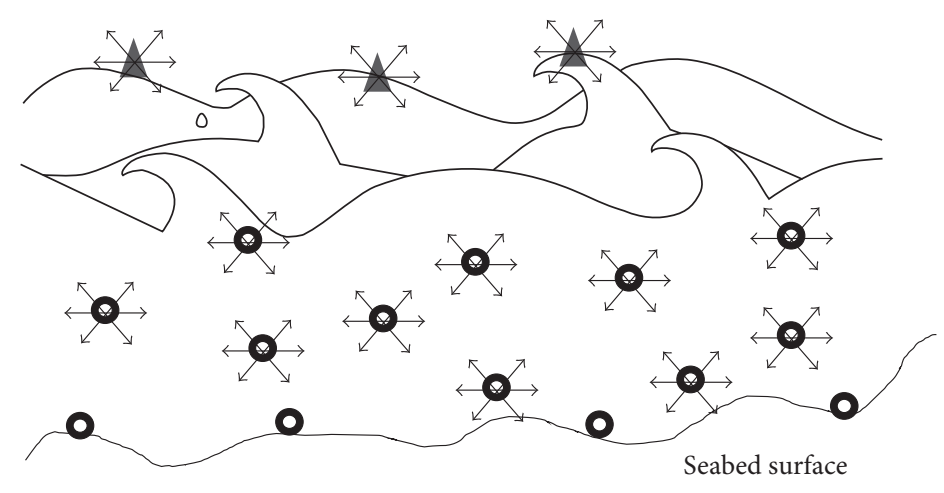

Floating sensor nodes
- Sloating sinks
- Static sensor nodes

FIGURE 2: Cyclic and irregular mobility patterns of nodes in an UWSN.

non-time-critical long-term (say, aquatic species monitoring) and time-critical short-term (say, submarine detection) applications. Secondly, if the deployed field is not under the designer's control or random deployment of the sensors is more feasible, stochastic deployment may be preferred [34]. In the latter case, an optimization problem on sensor deployment needs to be formulated to provide sufficient grid coverage of the sensor field such that maximum coverage may be achieved.

Due to the ocean current, UWSN nodes may move at speeds up to six kilometres an hour in a typical underwater scenario [3]. Hence, unlike most terrestrial sensor networks, where sensor nodes are mostly static, most sensor nodes placed underwater have slow to medium mobility. Consequently, any surveillance techniques designed by ignoring the mobility of sensor nodes may perform suboptimally when directly integrated into mobile UWSNs. It is important to note that the mobility models must be developed. This is due to the fact that the node mobility pattern in an UWSN is completely different from those usually considered in the above ground wireless sensor networks literature. The new mobility models have to be $3 \mathrm{D}$ in nature because of the cyclic or irregular patterns in forward and backward ways of ocean waves, as illustrated in Figure 2, something that is not the case in terrestrial networks.

Finally, the optimal architecture has to be of distributed types that takes the node depth into consideration with respect to the surface buoys. This way, the $3 \mathrm{D}$ coverage of the UWSN can be guaranteed. Therefore, the solutions should use adaptive node topology capable of adjusting the depth of sensor nodes in the event they drift by currents, winds, and so on.

3.2. Target Detection Considerations. Target detection deals with how the network detects or recognizes the presence of a mobile target in the proximity. As per the literature, a number of efforts have been made for underwater target detection, where most mechanisms have been based on the classical Doppler equation [35]. The primary motive behind using Doppler equation based techniques is due to the fact that most UWSNs were based on acoustic wave-based sensors. Further, the accuracy of the Doppler equation can only be guaranteed when the target moves to or from the listener, which means that there is a degree of inaccuracy involved in this method. Also, the node placement and the target characteristics must be known, which cannot be done for an underwater stochastically deployed environment. Therefore, due to the previously mentioned deficiencies of acoustic wave-based systems there are a number of prospects in using EM-based UWSNs.

In the case of stochastically deployed EM-based UWSNs, target detection can be achieved probabilistically. By the evaluation of the detection probability of at least $k$ sensors, the success of the target detection can be measured [34]. However, in more realistic scenarios, a sensor needs to collect multiple samples of the target before it can perform reliable detection $[36,37]$. Hence, a sensor $s$ must sample the target $X$ for at least $t$ units of time, before $s$ can reliably determine the presence of $X$. The most important point to be considered is that, since [34-37] are aboveground scenarios, underwater propagation characteristics of EM waves have to be taken into consideration in the development process.

3.3. Target Classification Considerations. Classification deals with how the network classifies the target type. Under this method, a classification-based data mining scheme can be implemented, where the system can classify the submerged targets as submarines, mines, divers, and sea animals. This is achieved with a combination of sensors based on radiation, mechanical, magnetic, thermal, and chemical signaling. In this particular case, the method that can be used for classification of targets is decision trees. The reason behind choosing decision trees is due to its fast executing, scalability, and ease of interpreting characteristics [38]. In order to use collaborative computing, classification-based data mining combined with the previous decision tree to detect and classify a target can be used. Each target type can be studied for defining the possible set of data values that a sensor could be measuring in the vicinity of a target. Next the challenge of 

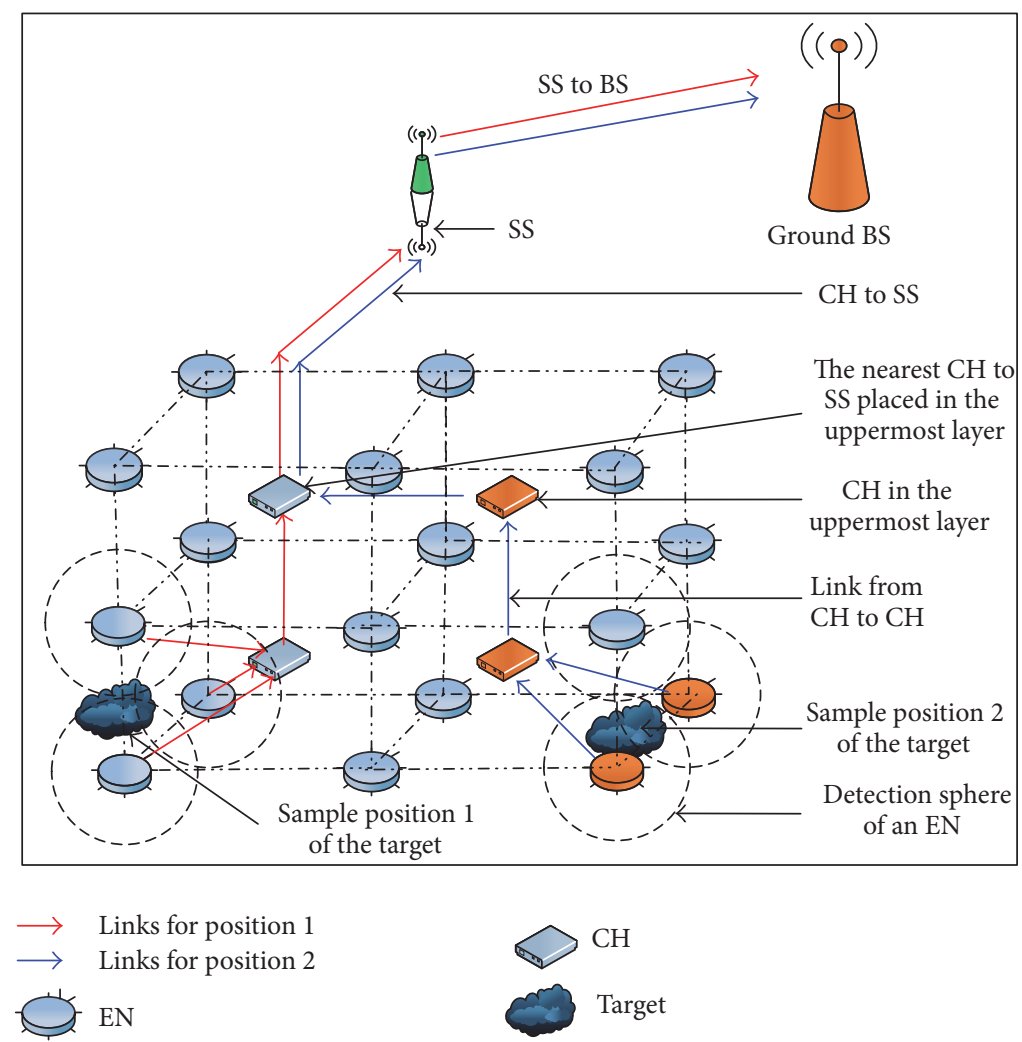

Figure 3: A general view of the proposed underwater target detection and tracking system.

preparation of the aforementioned data set for a classification mining based detection algorithm is involved.

3.4. Target Tracking Considerations. Once a target has been detected and classified, localization deals with sustained target tracking. As mentioned under Node Topology Design Considerations, a distributed tracking architecture is the preferred choice for UWSNs. In a potential distributed tacking architecture, the processing node must be chosen near the target and a subset of sensors in its vicinity are chosen as sensor data collection nodes. The moving target tracking problem can be handled as a multisensor data fusion problem. Under this, measurements from various sensors are combined and pull all information together as one coherent structure. Interacting multiple model (IMM) filters in this collaborative manoeuvring target tracking problem can also be used. Based on the received data, the IMM filter is capable of achieving its movement detection functionality by updating the mode probabilities making it advantageous over simpler estimators like Kalman filter [39]. Next the distributed IMM filter is used for estimating states of a target on a given sensor platform (assuming the target moves along a variety of trajectories). The distributed IMM filter then can combine different models as per the target's motion characteristics such that it will adapt to any change of trajectory and then the probability for each model can be calculated. Finally the complete target tracking model can be combined with an adaptive sensor selection scheme and an appropriate sleep/wake model for ensuring improved estimation and energy-efficient performance.

\section{EM Wave-Based Three-Dimensional UWSN Architectures for Localization and Target Tracking}

4.1. Basic Network Layout. We consider a 3D target surveillance area of dimension $D_{X} \times D_{Y} \times D_{Z}$, where $D_{X}, D_{Y}$, and $D_{Z}$ are the length of the surveillance area along the $X-, Y$ , and $Z$-axis, respectively. For the convenience, we assume a plane seabed. The $X-Y$ plane of the area is assumed parallel to the seabed and the $Z$-axis is along the depth of the sea. We propose grid-based network topologies by dividing the entire network into $N_{X}=D_{X} / \Delta_{X}, N_{Y}=D_{Y} / \Delta_{Y}$, and $N_{Z}=$ $D_{Z} / \Delta_{Z}$ segments along the three axes respectively, where $\Delta_{X}, \Delta_{Y}$, and $\Delta_{Z}$ are the corresponding segment lengths. The proposed UWSN topologies consist of two basic elements: the elementary nodes (ENs) which are essentially sensor nodes and the cluster heads (CHs). The general view of the overall target detection and tracking scheme as illustrated in Figure 3 consists of a UWSN, a surface sink (SS), and a ground base station (BS). When a target enters the surveillance area, some ENs sense the presence of the target and broadcast their location information to the surrounding $\mathrm{CH}$ s using EM wave. Upon receiving EM signal from ENs, nearby CHs collect different information and then forward the selected information to the respective $\mathrm{CH}$ s of immediate upper layer and so on. The information is subsequently routed to the $\mathrm{CH}$ nearest to the SS and finally to the SS. The SS then transmits the gathered information to the ground BS for processing and estimating the location and travelling path of the target. All the links 


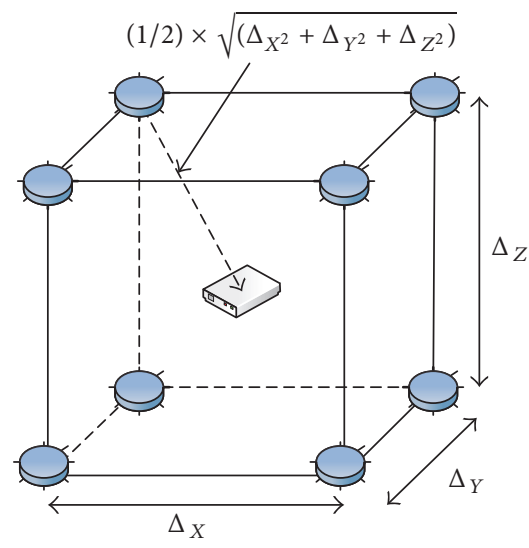

(a)

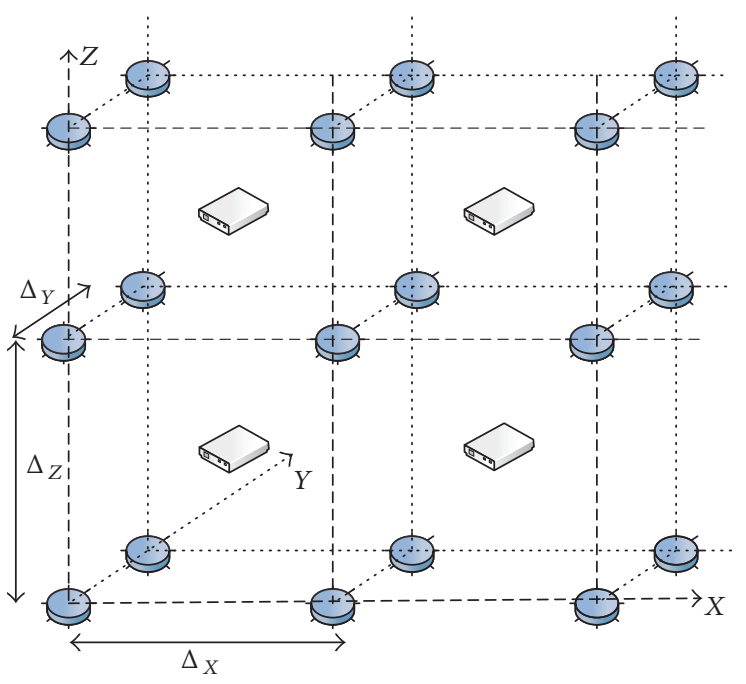

(b)

FIGURE 4: Proposed network topology for architecture $\mathrm{A}_{1}$ and $\mathrm{A}_{2}$ : (a) basic building block and (b) UWSN.

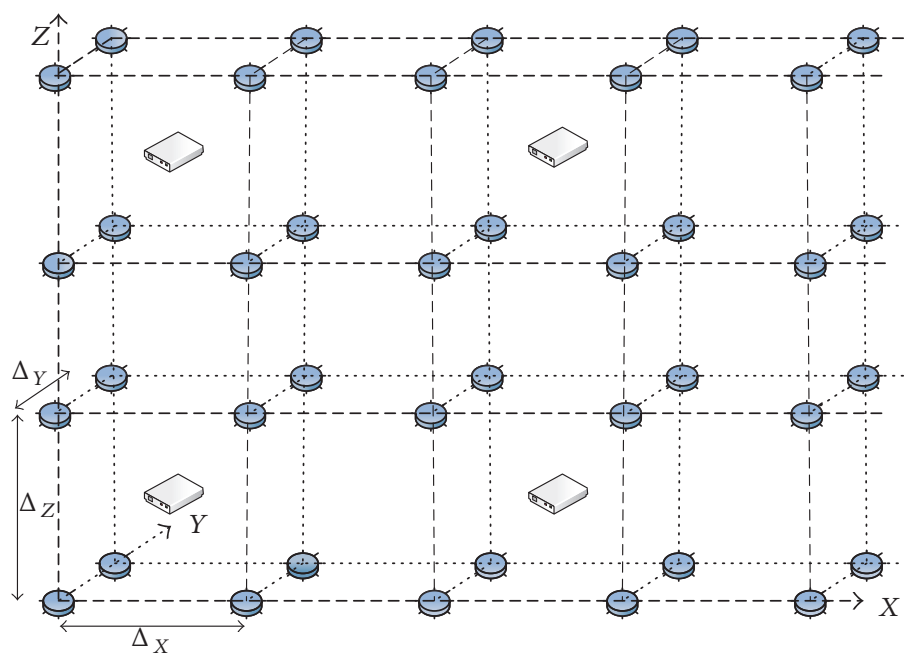

FIgURE 5: Proposed UWSN with architecture $A_{3}$ and $A_{5}$.

including $\mathrm{EN}-\mathrm{CH}, \mathrm{CH}-\mathrm{CH}, \mathrm{CH}-\mathrm{SS}$, and SS-BS are proposed to be EM wave-based.

4.2. Architectures. Under the proposed architectures, ENs and $\mathrm{CHs}$ are arranged in three different formations in $3 \mathrm{D}$ space evolving to three distinct basic building blocks of the UWSN. The basic building blocks of the topologies are repeated in $3 \mathrm{D}$ space to form the entire UWSNs covering the surveillance area of dimension $D_{X} \times D_{Y} \times D_{Z}$. The resulting three UWSNs using three distinct topologies are shown in Figures 4-6. The basic building block is shown only in Figure 4, which can similarly be drawn for the networks in Figures 5 and 6 . Now, based on these three distinct node topologies and different location estimation algorithms, we propose five different architectures denoted as $A_{1}, A_{2}, \ldots, A_{5}$ as presented below.
4.2.1. Architectures $A_{1}$ and $A_{2}$. The topology of the architectures $A_{1}$ and $A_{2}$ are same. However, the location estimation principles have significant difference. As shown in Figure 4(a), ENs are placed at all the eight vertices of a rectangular cuboid of dimension $\Delta_{X} \times \Delta_{Y} \times \Delta_{Z}$. Thus, the entire UWSN is formed by placing ENs every $\Delta_{X}, \Delta_{Y}$, and $\Delta_{Z}$ distance along $X$-, $Y$-, and $Z$-axis, respectively. On the other hand, one $\mathrm{CH}$ is placed at the centre of each cuboid. The UWSN thus has eight ENs surrounding each $\mathrm{CH}$ and vice versa. Thus the total number of CHs, $N_{\mathrm{CH}}$ and ENs, $N_{\mathrm{EN}}$ can be written as $N_{\mathrm{CH}}=N_{X} N_{Y} N_{Z}$ and $N_{\mathrm{EN}}=\left(N_{X}+1\right)\left(N_{Y}+1\right)\left(N_{Z}+1\right)$.

Now, for architecture $A_{1}$, we assume that each $\mathrm{CH}$ knows its own $3 \mathrm{D}$ coordinate. When an EN senses the presence of a target, it alerts the surrounding $\mathrm{CHs}$ by transmitting signal using EM wave. When a $\mathrm{CH}$ receives this EM signal with a power above a certain threshold $P_{\text {th }}$, it transmits its 3D coordinate to the immediate upper $\mathrm{CH}$ using EM wave. Through 


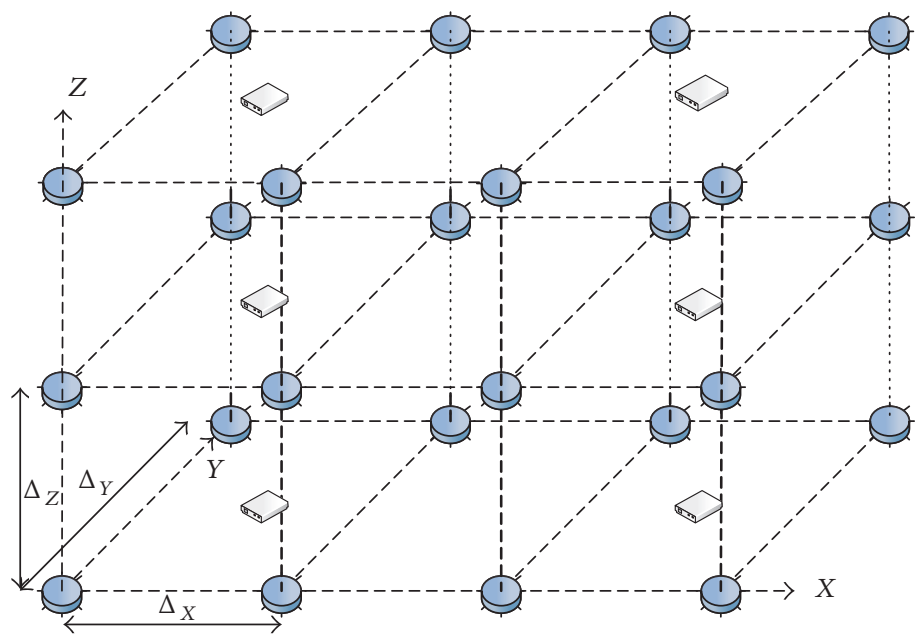

FIgURE 6: Proposed UWSN with architecture $\mathrm{A}_{4}$.

this flow of location data from one layer of $\mathrm{CH}$ s to those of the upper layers, the coordinates of the CHs nearest to the sensing ENs are known to the BS. Then the ground BS estimates the $3 \mathrm{D}$ coordinate $\left(x_{\text {est }}, y_{\text {est }}, z_{\text {est }}\right)$ of the target as follows:

$$
\begin{aligned}
& x_{\mathrm{est}}=\frac{1}{N} \sum_{i=1}^{N} x_{i}, \\
& y_{\mathrm{est}}=\frac{1}{N} \sum_{i=1}^{N} y_{i}, \\
& z_{\mathrm{est}}=\frac{1}{N} \sum_{i=1}^{N} z_{i},
\end{aligned}
$$

where $\left(x_{i}, y_{i}, z_{i}\right)$ is the $3 \mathrm{D}$ coordinate of the $i$ th $\mathrm{CH}$ and $N$ is the number of $\mathrm{CH}$ from which the ground $\mathrm{BS}$ has received information.

On the other hand, architecture $A_{2}$ utilizes both the location information of the $\mathrm{CH}$ s sending the target presence information to the ground BS as well as the EM signal power strength received from the surrounding ENs. The first step of estimating the target location is similar to architecture $A_{1}$. Thus, an initial estimate of the 3D target location denoted by $\left(x_{\text {int }}, y_{\text {int }}, z_{\text {int }}\right)$ is evaluated using (1). Then, by using the information of the received power at $\mathrm{CHs}$ from the surrounding ENs, the initially estimated location $\left(x_{\text {int }}, y_{\text {int }}, z_{\text {int }}\right)$ is finetuned to achieve a new coordinate, which can be given by

$$
\begin{aligned}
& x_{\mathrm{est}}=\frac{1}{N} \sum_{i=1}^{N} \frac{\left(P_{\max }-P_{i}\right) x_{\mathrm{int}}-\left(P_{\mathrm{int}}-P_{i}\right) x_{i}}{P_{\max }-P_{\mathrm{int}}}, \\
& y_{\mathrm{est}}=\frac{1}{N} \sum_{i=1}^{N} \frac{\left(P_{\max }-P_{i}\right) y_{\mathrm{int}}-\left(P_{\mathrm{int}}-P_{i}\right) y_{i}}{P_{\max }-P_{\mathrm{int}}}, \\
& z_{\mathrm{est}}=\frac{1}{N} \sum_{i=1}^{N} \frac{\left(P_{\max }-P_{i}\right) z_{\mathrm{int}}-\left(P_{\mathrm{int}}-P_{i}\right) z_{i}}{P_{\max }-P_{\mathrm{int}}},
\end{aligned}
$$

where $P_{i}$ is the actual EM power received at $i$ th $\mathrm{CH} ; P_{\max }$ and $P_{\text {int }}$ are the received power at $i$ th $\mathrm{CH}$ if ENs were located at the location of $\mathrm{CH}$ and $\left(x_{\text {int }}, y_{\text {int }}, z_{\text {int }}\right)$, respectively. All the values of power used in (2) are given in $\mathrm{dBm}$. Equation (2) is developed based on the fact that if we ignore fading, then the received power expressed in $\mathrm{dBm}$ decreases linearly with distance between the transmitter and the receiver.

4.2.2. Architectures $A_{3}$ and $A_{5}$. The topologies of architectures $\mathrm{A}_{3}$ and $\mathrm{A}_{5}$ (Figure 5) differ from those of architectures $A_{1}$ and $A_{2}$ (Figure 4) in the number and the locations of $\mathrm{CHs}$. As illustrated for architectures $\mathrm{A}_{3}$ and $\mathrm{A}_{5}, \mathrm{CH}$ are placed at the centre of every other rectangular cuboid of dimension $\Delta_{X} \times \Delta_{Y} \times \Delta_{Z}$ along all the three axes. Thus $\mathrm{CH}$ are placed every $2 \Delta_{X}, 2 \Delta_{Y}$, and $2 \Delta_{Z}$ distances along $X-, Y$-, and $Z$ axis, respectively. Hence, $N_{\mathrm{CH}}=\left\lceil N_{X} / 2\right\rceil\left\lceil N_{Y} / 2\right\rceil\left\lceil N_{Z} / 2\right\rceil$ and $N_{\mathrm{EN}}=\left(N_{X}+1\right)\left(N_{Y}+1\right)\left(N_{Z}+1\right)$. Here $\lceil x\rceil$ implies the ceiling operation and is equal to the smallest integer equal to or greater than $x$.

Now, similar to architecture $A_{1}$, architecture $A_{3}$ estimates the $3 \mathrm{D}$ location of a target from the location of the active $\mathrm{CHs}$ by using (1). On the other hand, although the topology of architecture $A_{5}$ is the same as that of $A_{3}, A_{5}$ integrates additional feature into the $\mathrm{CHs}$. It is assumed that each $\mathrm{CH}$ is equipped with eight directional receivers directed to the eight ENs surrounding it. So when a $\mathrm{CH}$ receives signal from surrounding ENs, it sorts out the strongest EM signal and the location of the corresponding EN from which the signal is received. Unlike $A_{1}-A_{3}$, instead of its own location, a $C H$ then transmits the location of this EN of the strongest EM signal to the $\mathrm{CH}$ of its upper layer and so on until it is received by the BS. The BS then estimates the location of the target using (1) replacing $x_{i}, y_{i}$, and $z_{i}$ by the coordinate of $i$ th EN contributing the strongest EM signal.

4.2.3. Architecture $A_{4}$. In the topology of architecture $\mathrm{A}_{4}$ as shown in Figure 6, though the placement of ENs is the same as all other architectures, $\mathrm{CH}$ are positioned in a very different way. Instead of placing CHs at the centre of cuboids, they are deployed on the $Z$-planes at the centre of four coplanar ENs. Thus, ENs are placed at regular intervals of $2 \Delta_{X}, 2 \Delta_{Y}$, and 
TABLE 2: A summary of the key features of the proposed architectures.

\begin{tabular}{|c|c|c|c|c|c|}
\hline Architectures & $\begin{array}{c}\text { Spacing between } \\
\text { two ENs }\end{array}$ & $\begin{array}{l}\text { Spacing between } \\
\text { two CHs }\end{array}$ & $\begin{array}{c}\text { Total number of ENs } \\
\text { and } \mathrm{CHs}\end{array}$ & $\begin{array}{l}\text { Directional } \\
\text { receiving antennas } \\
\text { in } \mathrm{CHs}\end{array}$ & $\begin{array}{c}\text { Information used } \\
\text { for location } \\
\text { estimation }\end{array}$ \\
\hline$A_{1}$ & $\Delta_{X}, \Delta_{Y}, \Delta_{Z}$ & $\Delta_{X}, \Delta_{Y}, \Delta_{Z}$ & $\begin{array}{c}N_{\mathrm{CH}}=N_{X} N_{Y} N_{Z} \\
N_{\mathrm{EN}}= \\
\left(N_{X}+1\right)\left(N_{Y}+1\right)\left(N_{Z}+1\right)\end{array}$ & No & Location of $\mathrm{CHs}$ \\
\hline $\mathrm{A}_{2}$ & $\Delta_{X}, \Delta_{Y}, \Delta_{Z}$ & $\Delta_{X}, \Delta_{Y}, \Delta_{Z}$ & $\begin{array}{c}N_{\mathrm{CH}}=N_{X} N_{Y} N_{Z} \\
N_{\mathrm{EN}}= \\
\left(N_{X}+1\right)\left(N_{Y}+1\right)\left(N_{Z}+1\right)\end{array}$ & No & $\begin{array}{l}\text { Location of CHs } \\
\text { and EM power } \\
\text { received at CHs }\end{array}$ \\
\hline $\mathrm{A}_{3}$ & $\Delta_{X}, \Delta_{Y}, \Delta_{Z}$ & $2 \Delta_{X}, 2 \Delta_{Y}, 2 \Delta_{Z}$ & $\begin{array}{c}N_{\mathrm{CH}}= \\
\left\lceil\frac{N_{X}}{2}\right\rceil\left\lceil\frac{N_{Y}}{2}\right\rceil\left\lceil\frac{N_{Z}}{2}\right\rceil \\
\left(N_{\mathrm{EN}}=1\right)\left(N_{Y}+1\right)\left(N_{Z}+1\right)\end{array}$ & No & Location of $\mathrm{CHs}$ \\
\hline $\mathrm{A}_{4}$ & $\Delta_{X}, \Delta_{Y}, \Delta_{Z}$ & $2 \Delta_{X}, 2 \Delta_{Y}, \Delta_{Z}$ & $\begin{array}{c}N_{\mathrm{CH}}= \\
\left\lceil\frac{N_{X}}{2}\right\rceil\left\lceil\frac{N_{Y}}{2}\right\rceil\left(N_{Z}+1\right) \\
N_{\mathrm{EN}}= \\
\left(N_{X}+1\right)\left(N_{Y}+1\right)\left(N_{Z}+1\right)\end{array}$ & $\begin{array}{c}\text { Four directional } \\
\text { antennas at each } \\
\mathrm{CH}\end{array}$ & $\begin{array}{l}\text { Location of ENs } \\
\text { contributing the } \\
\text { strongest EM } \\
\text { signal }\end{array}$ \\
\hline $\mathrm{A}_{5}$ & $\Delta_{X}, \Delta_{Y}, \Delta_{Z}$ & $2 \Delta_{X}, 2 \Delta_{Y}, 2 \Delta_{Z}$ & $\begin{array}{c}N_{\mathrm{CH}}= \\
\left.\left\lceil\frac{N_{X}}{2}\right\rceil \underset{N_{Y}}{2}\right\rceil\left\lceil\frac{N_{Z}}{2}\right\rceil \\
\left(N_{\mathrm{EN}}+1\right)\left(N_{Y}+1\right)\left(N_{Z}+1\right)\end{array}$ & $\begin{array}{c}\text { Eight directional } \\
\text { antennas at each } \\
\mathrm{CH}\end{array}$ & $\begin{array}{l}\text { Location of ENs } \\
\text { contributing the } \\
\text { strongest EM } \\
\quad \text { signal }\end{array}$ \\
\hline
\end{tabular}

$\Delta_{Z}$ distance along $X$-, $Y$-, and $Z$-axis, respectively, as shown in the figure. Hence, $N_{\mathrm{CH}}=\left\lceil N_{X} / 2\right\rceil\left\lceil N_{Y} / 2\right\rceil\left(N_{Z}+1\right)$ and $N_{\mathrm{EN}}$ is same as that of the previous architectures. Furthermore, each $\mathrm{CH}$ is equipped with four directional receivers directed to the four coplanar ENs surrounding it for determining the EN from which the strongest EM signal is received. Similar to architecture $\mathrm{A}_{5}$, the coordinate of the corresponding EN of the strongest EM signal is subsequently received by the BS and then used to estimate the location of target using (1). Here, $\left(x_{i}, y_{i}, z_{i}\right)$ is once again the coordinate of $i$ th EN contributing the strongest EM signal.

For the clarity, Table 2 summarizes the key features of the proposed underwater target detection and tracking architectures.

\subsection{Performance Metrics}

4.3.1. Error in Location Estimation. If $(x, y, z)$ is the actual location of a target, then the absolute distance $r$ between the actual and the estimated location becomes a metric for location estimation error and can be given by

$$
r=\sqrt{\left(x_{\mathrm{est}}-x\right)^{2}+\left(y_{\mathrm{est}}-y\right)^{2}+\left(z_{\mathrm{est}}-z\right)^{2}} .
$$

Then the normalized mean square error (NMSE) of location estimation defined as the mean of $r^{2}$ normalized by the square of the minimum distance between two ENs can be given by

$$
\mathrm{NMSE}_{D}=\frac{\sum_{i=1}^{M} r_{i}^{2}}{M\left[\min \left(\Delta_{x}, \Delta_{y}, \Delta_{z}\right)\right]^{2}},
$$

where $r_{i}$ is the location estimation error of $i$ th simulations and $M$ is the number of Monte Carlo simulations.

4.3.2. Error in Estimated Distance Travelled. If $\left(x_{1}, y_{1}, z_{1}\right)$ and $\left(x_{2}, y_{2}, z_{2}\right)$ are two different subsequent actual locations of a moving target and if the corresponding estimated locations of the target are $\left(x_{e 1}, y_{e 1}, z_{e 1}\right)$ and $\left(x_{e 2}, y_{e 2}, z_{e 2}\right)$, then the actual and the estimated travelled distance denoted by $r_{a}$ and $r_{e}$ can be calculated as

$$
\begin{aligned}
& r_{a}=\sqrt{\left(x_{1}-x_{2}\right)^{2}+\left(y_{1}-y_{2}\right)^{2}+\left(z_{1}-z_{2}\right)^{2}} \\
& r_{e}=\sqrt{\left(x_{e 1}-x_{e 2}\right)^{2}+\left(y_{e 1}-y_{e 2}\right)^{2}+\left(z_{e 1}-z_{e 2}\right)^{2}} .
\end{aligned}
$$

Then the NMSE of the estimated travelled distance can be defined as

$$
\mathrm{NMSE}_{T}=\frac{\sum_{i=1}^{M}\left(r_{i, a}-r_{i, e}\right)^{2}}{M\left[\min \left(\Delta_{x}, \Delta_{y}, \Delta_{z}\right)\right]^{2}},
$$

where $r_{i, a}$ and $r_{i, e}$ are the actual and the estimated travelled distance respectively of $i$ th simulations.

4.3.3. Error in Travelled Direction Estimation. Error in estimated horizontal direction of travelling denoted as $\theta_{X Y}$ and error in estimated vertical direction of travelling denoted as $\theta_{Y Z}$ can be defined as follows:

$$
\begin{aligned}
& \theta_{X Y}=\tan ^{-1}\left(\frac{y_{e 1}-y_{e 2}}{x_{e 1}-x_{e 2}}\right)-\tan ^{-1}\left(\frac{y_{1}-y_{2}}{x_{1}-x_{2}}\right), \\
& \theta_{Y Z}=\tan ^{-1}\left(\frac{z_{e 1}-z_{e 2}}{y_{e 1}-y_{e 2}}\right)-\tan ^{-1}\left(\frac{z_{1}-z_{2}}{y_{1}-y_{2}}\right) .
\end{aligned}
$$


Then the root mean square error (RMSE) in the estimated direction of travelling can be defined as

$$
\begin{aligned}
\operatorname{RMSE}_{X Y} & =\sqrt{\frac{1}{M} \sum_{i=1}^{M} \theta_{i, X Y}^{2}}, \\
\mathrm{RMSE}_{Y Z} & =\sqrt{\frac{1}{M} \sum_{i=1}^{M} \theta_{i, Y Z}^{2}}
\end{aligned}
$$

4.3.4. Propagation Delay. Propagation delay of a communication system can be evaluated by dividing the total distance a signal travel with the propagation speed of the signal. The propagation speed of EM wave in underwater environment can be represented by the following equation [12]:

$$
C_{w}=\sqrt{\frac{f \times 10^{7}}{\sigma}}, \mathrm{m} / \mathrm{s},
$$

where $f$ is the transmission frequency in $\mathrm{Hz}$ and $\sigma$ is the conductivity of water in $\mathrm{S} / \mathrm{m}$. Typical value for $\sigma=0.01 \mathrm{~S} / \mathrm{m}$ for fresh water, which is much higher for seawater due to the higher salinity [12]. On the other hand, speed of acoustic signal in water can be approximately taken equal to $1,500 \mathrm{~m} / \mathrm{s}$ [12].

4.4. Performance Evaluation. We evaluate the performance of the proposed target detection and tracking architectures using MATLAB based Monte Carlo simulation platform. The results presented in this section are obtained through averaging over $M=10,000$ independent simulations. Performance evaluations are carried out considering only the underwater part of the complete surveillance system, which implies that any information reaching the SS will reach the ground BS as well. The parameters used for the simulations are chosen in reference to various references of RF based underwater communications $[12,40-42]$. Without losing the generality, a 3D UWSN with $D_{X}=D_{Y}=D_{Z}=D$ and $\Delta_{X}=$ $\Delta_{Y}=\Delta_{Z}=\Delta$ is considered for simulations. An underwater EM wave propagation model with path loss $20 \times\left(\log _{10} e\right) \times$ $2 \pi \times \sqrt{\left(\sigma \times f \times 10^{-7}\right)} \mathrm{dB} / \mathrm{m}$ and shadow fading is used [12]. Shadow fading is modeled as log-normally distributed random variable with a mean and standard deviation equal to $0 \mathrm{~dB}$ and $\zeta \mathrm{dB}$, respectively. Conductivity $\sigma=4 \mathrm{~S} / \mathrm{m}$ is used for emulating a typical salty sea water environment $[12,40]$. Transmission frequency equal to $6 \mathrm{kHz}$ and $3 \mathrm{kHz}$ is used for ENs and CHs, respectively [12, 41]. On the other hand, transmit power of EN and $\mathrm{CH}$ is assumed equal to $100 \mathrm{~mW}$ and $1 \mathrm{~W}$, respectively [42]. Unless otherwise stated, a network with $D=400 \mathrm{~m}, \Delta=20 \mathrm{~m}$, and $P_{\text {th }}=-60 \mathrm{dBm}$ is considered for the simulations.

4.4.1. Hardware Requirement. Figure 7 compares the architectures in terms of the number of $\mathrm{CH}$ ser EN considering an infinitely long UWSN with an infinitely large number of segments along the three axes. The ratios will be smaller for smaller sized networks and thus the figure represents the

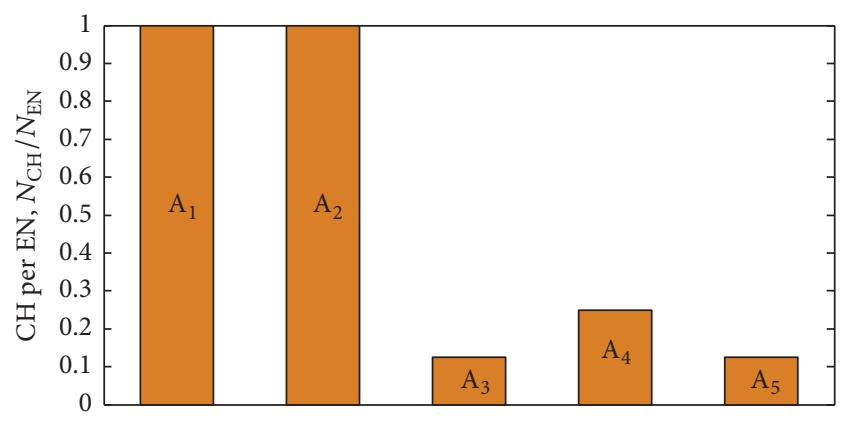

FIGURE 7: Number of CHs per EN for an infinitely long UWSN with an infinitely large number of segments along all the three axes (i.e., $N_{X} \rightarrow \infty, N_{Y} \rightarrow \infty$, and $\left.N_{Z} \rightarrow \infty\right)$.

worst case scenario requiring the maximum number of $\mathrm{CHs}$ per EN. Although the number of ENs in the total surveillance area is equal for all the five architectures, $\mathrm{CHs}$ are placed more sparsely in $A_{3}, A_{4}$ and $A_{5}$ compared to $A_{1}$ and $A_{2}$. This leads to the lower number of $\mathrm{CH}$ s per $\mathrm{EN}$ for the former three architectures.

4.4.2. Accuracy in Localizing Objects. Performance of the target detection architectures in terms of NMSE of the estimated location for two different detection thresholds with and without shadow fading is illustrated in Figure 8. Normalization is done using $\Delta=20 \mathrm{~m}$ and shadow fading is simulated using $\zeta=8 \mathrm{~dB}$. Network length is increased by keeping the relative positions and the number of ENs and $\mathrm{CH}$ s the same as those of a UWSN with $D=400 \mathrm{~m}$ and $\Delta=20 \mathrm{~m}$ leading to increased distances among the nodes in the networks. Several insights can now be identified by observing Figure 8. Firstly, the figure clearly shows increasing trends of NMSE of all the architectures with the increase of the network length. With the increased distance between any two nodes, fewer number of ENs can communicate with the corresponding $\mathrm{CH}$ and fewer $\mathrm{CH}$ s can communicate with the $\mathrm{CH}$ in the upper layer leading to reduced accuracy and increased NMSE. Secondly, the maximum network size is much smaller $\left(630 \mathrm{~m}\right.$ for $P_{\text {th }}=$ $-90 \mathrm{dBm})$ for $A_{3}$ and $A_{5}$ than that for $A_{1}, A_{2}$, and $A_{4}(1275 \mathrm{~m}$ for $\left.P_{\text {th }}=-90 \mathrm{dBm}\right)$, which is the direct result of larger distance between adjacent $\mathrm{CHs}$ in $\mathrm{A}_{3}$ and $\mathrm{A}_{5}$. Furthermore, comparing Figures 8(a)-8(c), it is found that with the increase of detection threshold from $-90 \mathrm{dBm}$ to $-30 \mathrm{dBm}$, the maximum network size is reduced from $1280 \mathrm{~m}$ to $640 \mathrm{~m}$ for $\mathrm{A}_{1}$, $\mathrm{A}_{2}$, and $\mathrm{A}_{4}$ and below $400 \mathrm{~m}$ for $\mathrm{A}_{3}$ and $\mathrm{A}_{5}$. Thirdly, the deteriorating impact of shadow fading on the performance of the architectures in terms of increased NMSE is clearly demonstrated in the figure. However, up to a certain network size, the performance of the architectures with and without fading environment is the same, which is due to the close proximity of network nodes such that the received signal power is above the detection threshold even in the presence of severe fading. Beyond this certain network size (around 1140$1170 \mathrm{~m}$ for $\mathrm{A}_{1}, \mathrm{~A}_{2}$, and $\mathrm{A}_{4}$ ), the performance gap between the two scenarios increases sharply. Moreover, as seen in Figures $8(\mathrm{a})-8(\mathrm{~b})$, the impact of shadow fading on the performance of $A_{3}$ and $A_{5}$ is not visible. This is due to the much lower 


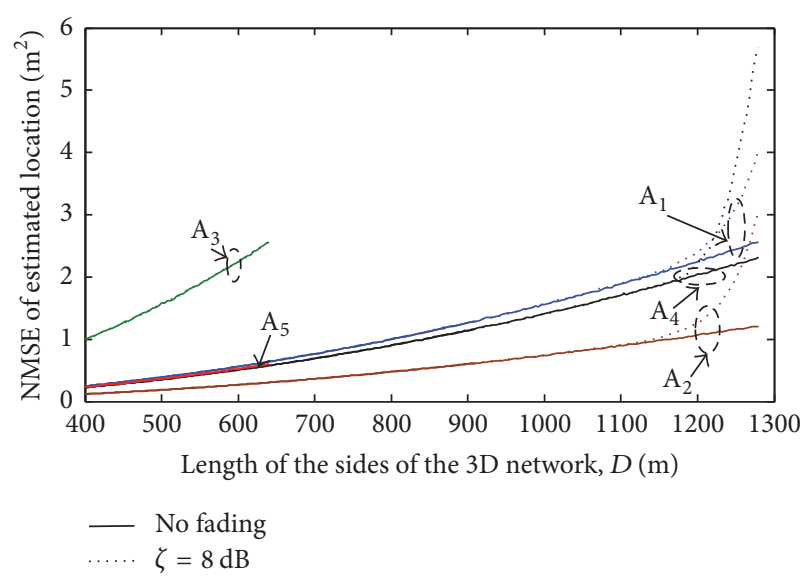

(a) Detection threshold $-90 \mathrm{dBm}$

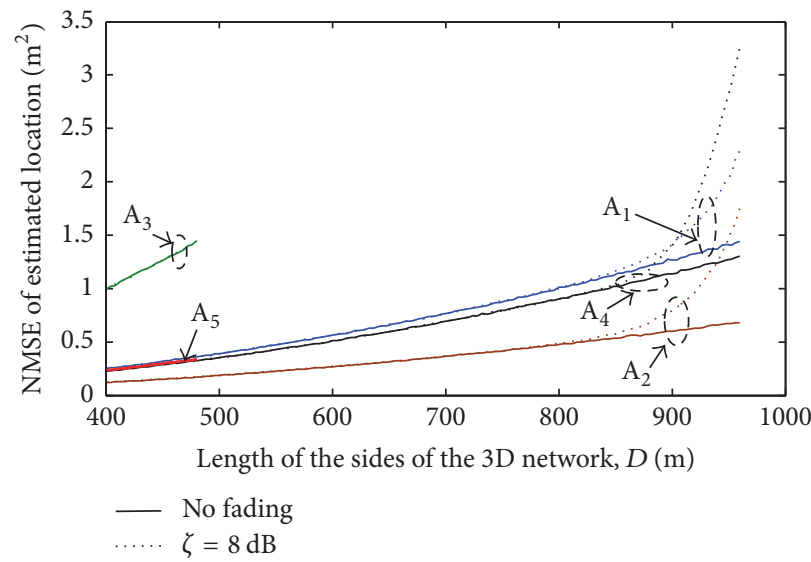

(b) Detection threshold $-60 \mathrm{dBm}$

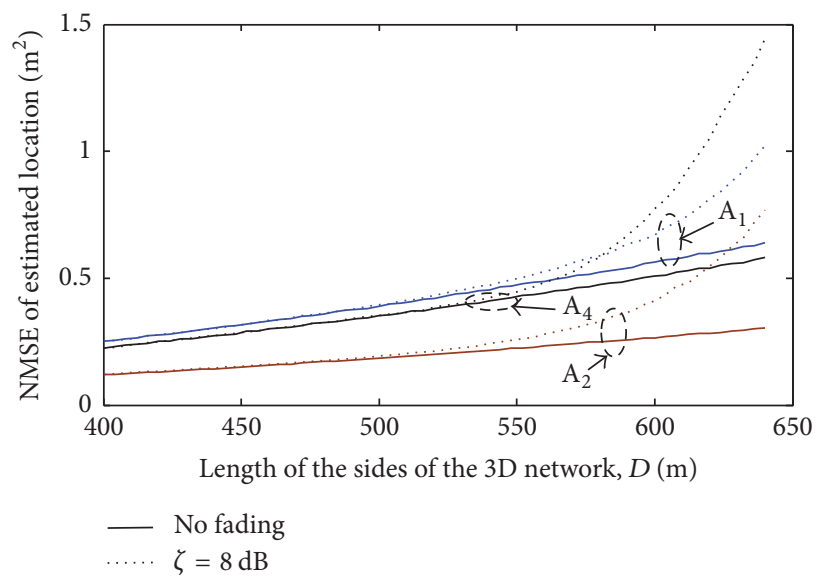

(c) Detection threshold $-30 \mathrm{dBm}$

FIGURE 8: NMSE of the estimated location of a target with network size keeping the number of networking nodes unchanged.

maximum network size $\left(630 \mathrm{~m}\right.$ for $P_{\mathrm{th}}=-90 \mathrm{dBm}$ and $475 \mathrm{~m}$ for $P_{\text {th }}=-60 \mathrm{dBm}$ ) compared to the other architectures up to which shadow fading does not degrade the received power by the network nodes to a value lower than $P_{\text {th }}$. Finally, it can readily be identified that the architecture $A_{3}$ has the worst accuracy, which is primarily due to the sparse location of $\mathrm{CHs}$, whereas the best accuracy is achieved for $\mathrm{A}_{2}$. It is also evident that with much fewer $\mathrm{CHs}, \mathrm{A}_{4}$ and $\mathrm{A}_{5}$ have improved performance than that of $A_{1}$. The reason behind this better accuracy is the use of directional antennas integrated in the $\mathrm{CHs}$ and the position estimations from the locations of ENs. However, this additional feature may increase the size, computational complexity, and energy consumption in $\mathrm{CHs}$. On the other hand, despite the use of the same topology, the better accuracy of $A_{2}$ compared to that of $A_{1}$ is directly attributed to the further fine-tuning of the initially estimated location using the information of received power. Thus it can be inferred that if the information of received power is utilized, the accuracy of $\mathrm{A}_{4}$ and $\mathrm{A}_{5}$ would be significantly improved, which can be further investigated.

4.4.3. Accuracy in Tracking Objects. On the other hand, for demonstrating the performance of the proposed architectures in tracking moving targets, a sample path of the intruder and the corresponding tracked path by $\mathrm{A}_{2}$ and $\mathrm{A}_{3}$ under no fading environment are illustrated in Figures 9(a)-9(b). From the visual inspection, it is clear that architecture $A_{2}$ has better accuracy than that of $\mathrm{A}_{3}$ in tracking the path of the target, which is also supported by Figures $8(\mathrm{a})-8(\mathrm{~b})$. On the other hand, the NMSE of the estimated travelled path and the RMSE of the angles in the $X Y$ and $Y Z$ planes with and without shadow fading are illustrated in Figure 10. For understanding the impact of shadow fading, the network is configured using $D=900 \mathrm{~m}$ (correspondingly $\Delta=45 \mathrm{~m}$ ). The figure does not include the results of $\mathrm{A}_{3}$ and $\mathrm{A}_{5}$ as $900 \mathrm{~m}$ is well above the feasible network size of these two architectures. A detection threshold $P_{\text {th }}=-60 \mathrm{dBm}$ is considered for the simulations. From the figure, $A_{2}$ is found to have the best accuracy in both estimating travelled distance and the travelled direction as evident from the plot of NMSE and RMSE, respectively, which is also supported by Figure 8 . Shadow fading can significantly reduce the accuracy in travelled distance estimation as evident from Figure 10(a). However, though shadow fading has little impact on the accuracy in estimating the travelled direction in $A_{4}$, negligible impact is seen in $A_{1}$ 


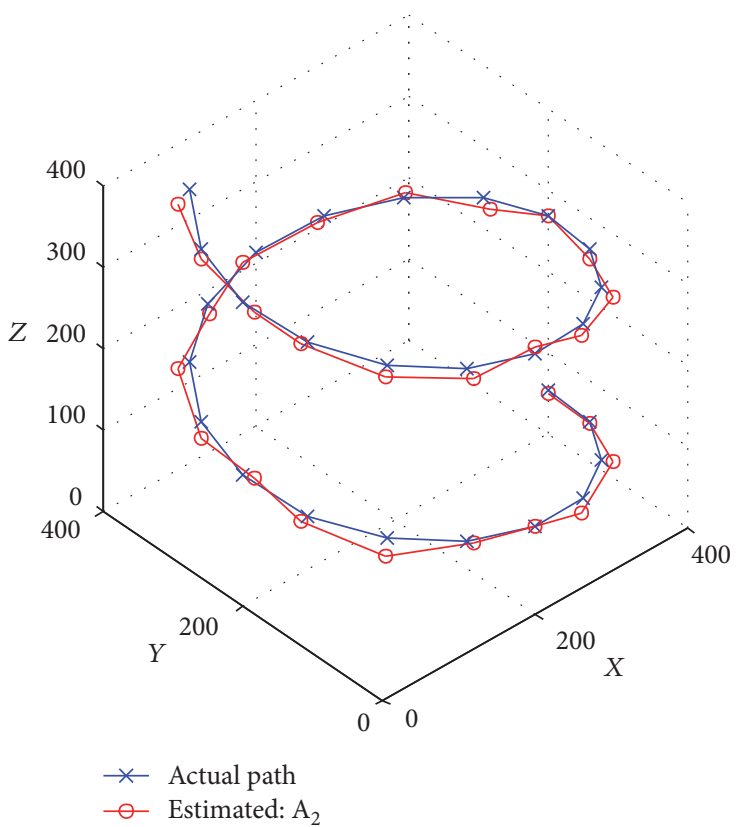

(a) Architecture $\mathrm{A}_{2}$

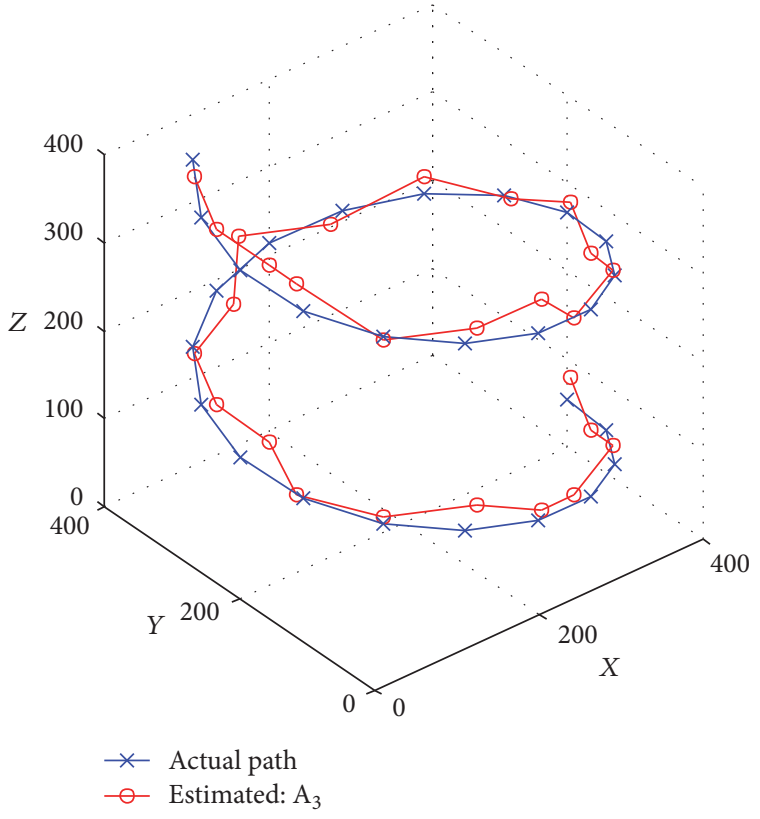

(b) Architecture $\mathrm{A}_{3}$

FIGURE 9: Sample travel path and the estimated path of a moving target with no fading.

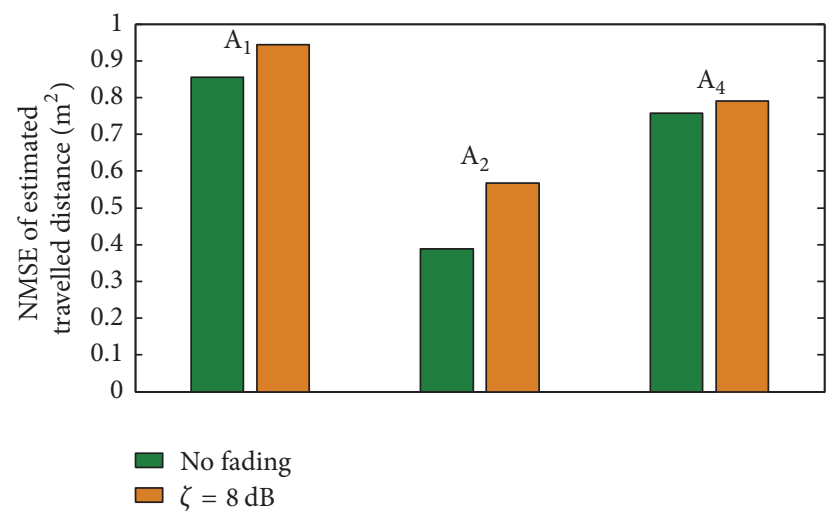

(a) NMSE of the estimation of travelled path

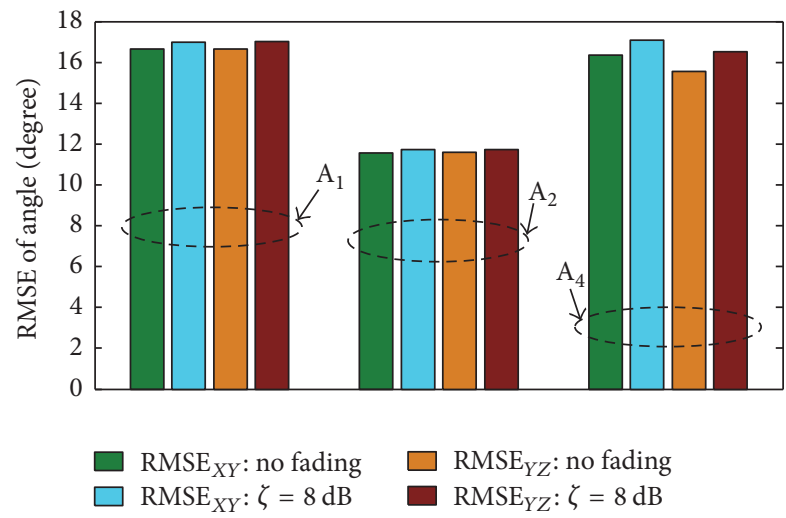

(b) RMSE of angles in $X Y$ and $Y Z$ planes

FIGURE 10: Performance of the architectures in tracking a moving target with $P_{\text {th }}=-60 \mathrm{dBm}$.

and $\mathrm{A}_{2}$ because of the symmetric nature of their network topology.

4.4.4. Response Time. Finally, Figures 11(a)-11(b) present the cumulative distribution function $(\mathrm{CDF})$ of the propagation time of signal from the sensing ENs to the SS for both the EM and acoustic wave-based systems. It is clearly seen that the propagation delay for EM wave-based system is of several orders of magnitude smaller than that in an acoustic wavebased one leading to fast intruder detection. The reason behind this significantly lower navigation delay of EM-based system is the fundamental characteristic of high propagation speed of EM waves compared to that of acoustic waves.

4.4.5. Nodes Mobility. For evaluating the performance of the proposed architectures, we have considered that the nodes are static with respect to each other. If we consider the relative displacement of nodes at their positions, accuracy of the proposed architectures will degrade and maximum allowable network size will decrease. That is, the performance will be affected in a similar way of shadow fading. Nevertheless, the proposed algorithms are equally applicable for mobile node scenario as well. It is worthwhile to mention that the proposed architectures with fixed node positions are also suitable for many practical applications where nodes mobility can be ignored and the relative displacement of the nodes are negligible. Such applications include 3D fence around sea beaches for detecting and tracking sharks, seaports and harbor for surveillance, offshore gas rigs, a moving grid, or fence fixed with a moving vessel. However, mobility of networking nodes is a critical issue for underwater networking, which depends on many factors including the water current patterns, moving 


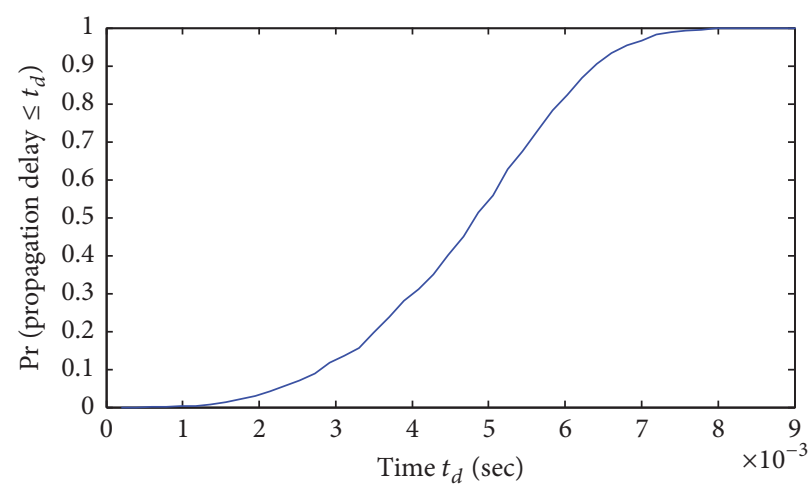

(a) EM wave-based

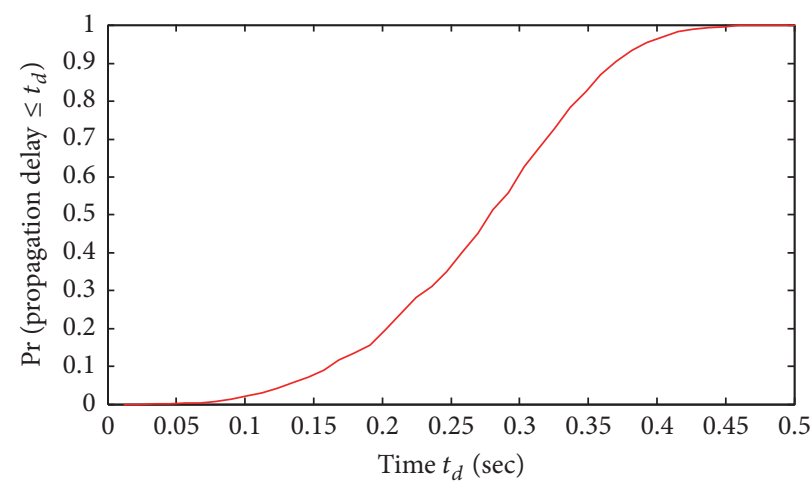

(b) Acoustic wave-based

FIGURE 11: CDF of propagation delays from EN to SS.

ships and vessels, and movement of underwater objects (e.g., fish, AUV, and submarines). Advanced techniques can be integrated with the proposed systems for overcoming performance degradation due to node mobility, which is left for future works.

\section{Conclusions}

There is a great demand for highly sophisticated yet economically viable solutions for underwater surveillance and exploration of maritime resources. In terms of underwater target tracking, UWSNs are considered as a tangible, low cost solution. Existing acoustic wave-based UWSN systems fail to meet today's growing demand for fast response and higher data rates. EM wave-based communication systems on the other hand have great potential for providing such requirements. This paper investigates the challenges of using EM wave-based UWSNs and evaluates several EM wave-based UWSN architectures on the performance of localization and target tracking. For nations that border the ocean, the need for faster and smarter underwater communication networks becomes even more critical. For instance, from industry, military, scientific, and environmental points of view, it is extremely vital to have fast, robust, scalable, and adaptive underwater communications. In our future works, we will focus on developing efficient node deployment strategies with different objectives for EM wave-based UWSNs for underwater target localization and tracking. These strategies will integrate sophisticated techniques for improving network robustness with mobile nodes, multipath propagation, and water dynamics.

\section{Competing Interests}

The authors declare that they have no competing interests.

\section{References}

[1] J. Partan, J. Kurose, and B. N. Levine, "A survey of practical issues in underwater networks," in Proceedings of the 1st ACM International Workshop on Underwater Networks (WUWNet '06), pp. 17-24, Los Angeles, Calif, USA, September 2006.
[2] R. Headrick and L. Freitag, "Growth of underwater communication technology in the U.S. Navy," IEEE Communications Magazine, vol. 47, no. 1, pp. 80-82, 2009.

[3] J.-H. Cui, J. Kong, M. Gerla, and S. Zhou, "The challenges of building scalable mobile underwater wireless sensor networks for aquatic applications," IEEE Network, vol. 20, no. 3, pp. 12-18, 2006.

[4] L. Liu, S. Zhou, and J.-H. Cui, "Prospects and problems of wireless communication for underwater sensor networks," Wireless Communications and Mobile Computing, vol. 8, no. 8, pp. 977-994, 2008.

[5] M. Asif, M. Rizal, and A. Yahya, "An active contour for underwater target tracking and navigation," in Proceedings of the International Conference on Man-Machine Systems, pp. 1-6, Langkawi Islands, Malaysia, September 2006.

[6] E. Dalberg, A. Lauberts, R. K. Lennartsson, M. J. Levonen, and L. Persson, "Underwater target tracking by means of acoustic and electromagnetic data fusion," in Proceedings of the 9th International Conference on Information Fusion (FUSION '06), July 2006.

[7] M. I. Pettersson, V. Zetterberg, and I. Claesson, "Detection and imaging of moving targets in wideband as using fast time back projection combined with space-time processing," in Proceedings of the MTS/IEEE Oceans, pp. 2388-2393, Washington, DC, USA, September 2005.

[8] Q. Zhang, M. Liu, S. Zhang, and H. Chen, "Node topology effect on target tracking based on underwater wireless sensor networks," in Proceedings of the 17th International Conference on Information Fusion (FUSION '14), Salamanca, Spain, July 2014.

[9] S. Al-Dharrab, M. Uysal, and T. Duman, "Cooperative underwater acoustic communications," IEEE Communications Magazine, vol. 51, no. 7, pp. 146-153, 2013.

[10] P. Casari and M. Zorzi, "Protocol design issues in underwater acoustic networks," Computer Communications, vol. 34, no. 17, pp. 2013-2025, 2011.

[11] J. G. Proakis, E. M. Sozer, J. A. Rice, and M. Stojanovic, "Shallow water acoustic networks," IEEE Communications Magazine, vol. 39, no. 11, pp. 114-119, 2001.

[12] X. Che, I. Wells, G. Dickers, P. Kear, and X. Gong, "Reevaluation of RF electromagnetic communication in underwater sensor networks," IEEE Communications Magazine, vol. 48, no. 12, pp. 143-151, 2010. 
[13] K. Chen, M. Ma, E. Cheng, F. Yuan, and W. Su, "A survey on MAC protocols for underwater wireless sensor networks," IEEE Communications Surveys and Tutorials, vol. 16, no. 3, pp. 14331447, 2014.

[14] G. A. Shah, "A survey on medium access control in underwater acoustic sensor networks," in Proceedings of the International Conference on Advanced Information Networking and Applications Workshops (WAINA '09), pp. 1178-1183, May 2009.

[15] I. F. Akyildiz, D. Pompili, and T. Melodia, "State of the art in protocol research for underwater acoustic sensor networks," ACM SIGMOBILE Mobile Computing and Communications Review, vol. 11, no. 4, pp. 11-22, 2007.

[16] D. Pompili, T. Melodia, and I. F. Akyildiz, "Deployment analysis in underwater acoustic wireless sensor networks," in Proceedings of the First ACM International Workshop on Underwater Networks ( WUWNet '06), pp. 48-55, Los Angeles, Calif, USA, September 2006.

[17] M. Arik and O. B. Akan, "Collaborative mobile target imaging in UWB wireless radar sensor networks," IEEE Journal on Selected Areas in Communications, vol. 28, no. 6, pp. 950-961, 2010.

[18] A. O. Bicen, A. B. Sahin, and O. B. Akan, "Spectrum-aware underwater networks: cognitive acoustic communications," IEEE Vehicular Technology Magazine, vol. 7, no. 2, pp. 34-40, 2012.

[19] I. F. Akyildiz, D. Pompili, and T. Melodia, "Underwater acoustic sensor networks: research challenges," Ad Hoc Networks, vol. 3, no. 3, pp. 257-279, 2005.

[20] J. Heidemann, W. Ye, J. Wills, A. Syed, and Y. Li, "Research challenges and applications for underwater sensor networking," in Proceedings of the IEEE Wireless Communications and Networking Conference (WCNC '06), vol. 4, pp. 228-235, Las Vegas, Nev, USA, April 2006.

[21] Q. Zhang, C. Zhang, M. Liu, and S. Zhang, "Local node selection for target tracking based on underwater wireless sensor networks," International Journal of Systems Science, vol. 46, no. 16, pp. 2918-2927, 2015.

[22] M. Erol, L. F. M. Vieira, and M. Gerla, "AUV-aided localization for underwater sensor networks," in Proceedings of the 2nd Annual International Conference on Wireless Algorithms, Systems, and Applications (WASA '07), pp. 44-54, IEEE, Chicago, Ill, USA, August 2007.

[23] J. E. Faugstadmo, "Underwater wireless sensor networks," in Proceedings of the 4th International Conference on Sensor Technologies and Applications (SENSORCOMM '10), Venice, Italy, July 2010.

[24] S. Zhou and P. Willett, "Submarine location estimation via a network of detection-only sensors," IEEE Transactions on Signal Processing, vol. 55, no. 6, pp. 3104-3115, 2007.

[25] Q. Liang and X. Cheng, "Underwater acoustic sensor networks: target size detection and performance analysis," Ad Hoc Networks, vol. 7, no. 4, pp. 803-808, 2009.

[26] Y. Huang, W. Liang, H.-B. Yu, and Y. Xiao, "Target tracking based on a distributed particle filter in underwater sensor networks," Wireless Communications and Mobile Computing, vol. 8, no. 8, pp. 1023-1033, 2008.

[27] X. Wang, M. Xu, H. Wang, Y. Wu, and H. Shi, "Combination of interacting multiple models with the particle filter for threedimensional target tracking in underwater wireless sensor networks," Mathematical Problems in Engineering, vol. 2012, Article ID 829451, 16 pages, 2012.

[28] M. T. Isik and O. B. Akan, "A three dimensional localization algorithm for underwater acoustic sensor networks," IEEE
Transactions on Wireless Communications, vol. 8, no. 9, pp. 4457-4463, 2009.

[29] C. Yu, K. Lee, J. Choi, and Y. Seo, "Distributed single target tracking in underwater wireless sensor networks," in Proceedings of the SICE Annual Conference, pp. 1351-1356, Tokyo, Japan, August 2008.

[30] P. Djukic, Y. Zhou, and M. Toulgoat, "Localization for electromagnetic radio underwater sensor networks," in Proceedings of the 5th International Conference on Sensor Technologies and Applications (SENSORCOMM '11), pp. 172-177, Nice, France, August 2011.

[31] Y. Zhu, X. Lu, L. Pu et al., "Aqua-Sim: an NS-2 based simulator for underwater sensor networks," in Proceedings of the ACM International Conference on Underwater Networks and Systems, pp. 1-2, Kaohsiung, Taiwan, November 2013.

[32] S. M. N. Alam and Z. J. Haas, "Coverage and connectivity in three-dimensional networks," in Proceedings of the 12th Annual International Conference on Mobile Computing and Networking (MOBICOM '06), pp. 346-357, Ithaca, NY, USA, September 2006.

[33] G. Han, C. Zhang, L. Shu, N. Sun, and Q. Li, "A survey on deployment algorithms in underwater acoustic sensor networks," International Journal of Distributed Sensor Networks, vol. 2013, Article ID 314049, 11 pages, 2013.

[34] Q. Cao, T. Yan, T. Abdelzaher, and J. Stankovic, "Analysis of target detection performance for wireless sensor networks," in Proceedings of the International Conference on Distributed Computing in Sensor Networks, Los Angeles, Calif, USA, June 2005.

[35] A. M. Mahdy and J. M. Groenke, "Target tracking in marine wireless sensor networks," International Journal on Advances in Networks and Services, vol. 3, no. 1-2, pp. 103-113, 2010.

[36] C. Gui and P. Mohapatra, "Power conservation and quality of surveillance in target tracking sensor networks," in Proceedings of the Tenth Annual International Conference on Mobile Computing and Networking (MobiCom '04), pp. 129-143, Philadelphia, Pa, USA, October 2004.

[37] A. Arora, P. Dutta, S. Bapat et al., "A line in the sand: a wireless sensor network for target detection, classification, and tracking," Computer Networks, vol. 46, no. 5, pp. 605-634, 2004.

[38] A. K. H. Tung, H. Lu, J. Han, and L. Feng, "Efficient mining of intertransaction association rules," IEEE Transactions on Knowledge and Data Engineering, vol. 15, no. 1, pp. 43-56, 2003.

[39] E. Mazor, "Interacting multiple model methods in target tracking: a survey," IEEE Transactions on Aerospace and Electronic Systems, vol. 34, no. 1, pp. 103-123, 1998.

[40] A. I. Al-Shamma'a, A. Shaw, and S. Saman, "Propagation of electromagnetic waves at $\mathrm{MHz}$ frequencies through seawater," IEEE Transactions on Antennas and Propagation, vol. 52, no. 11, pp. 2843-2849, 2004.

[41] I. Wells, A. Davies, X. Che et al., "Node pattern simulation of an undersea sensor network using RF electromagnetic communications," in Proceedings of the IEEE International Conference on Ultra Modern Telecommunications and Workshops (ICUMT '09), pp. 1-4, IEEE, St. Petersburg, Russia, October 2009.

[42] M. R. Frater, M. J. Ryan, and R. M. Dunbar, "Electromagnetic communications within swarms of autonomous underwater vehicles," in Proceedings of the First ACM International Workshop on Underwater Networks (WUWNet '06), pp. 64-70, Los Angeles, Calif, USA, September 2006. 


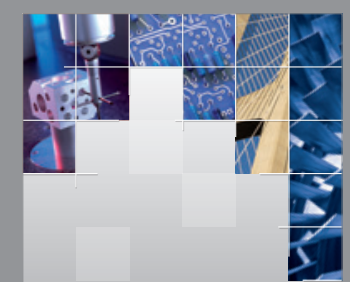

\section{Enfincering}
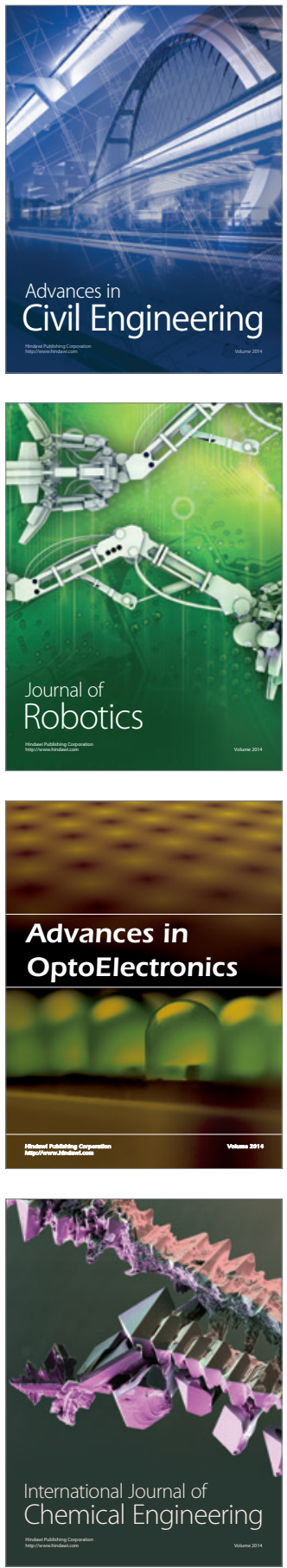

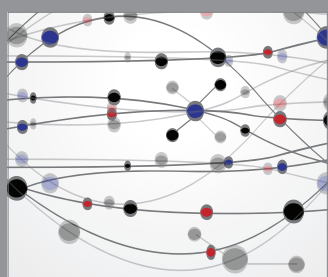

The Scientific World Journal

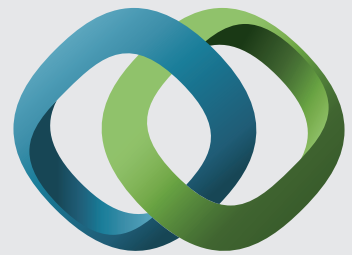

\section{Hindawi}

Submit your manuscripts at

https://www.hindawi.com
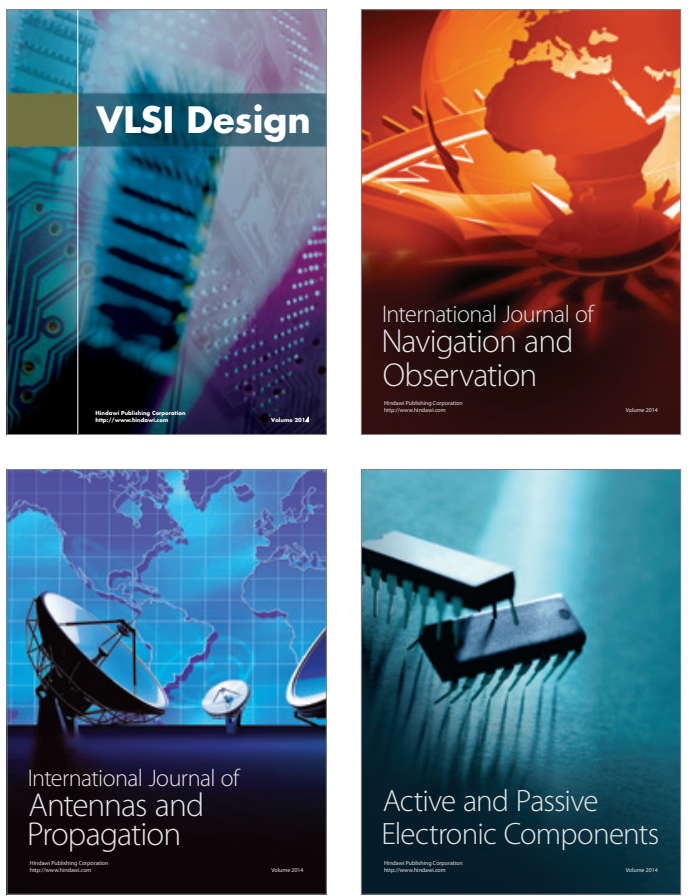
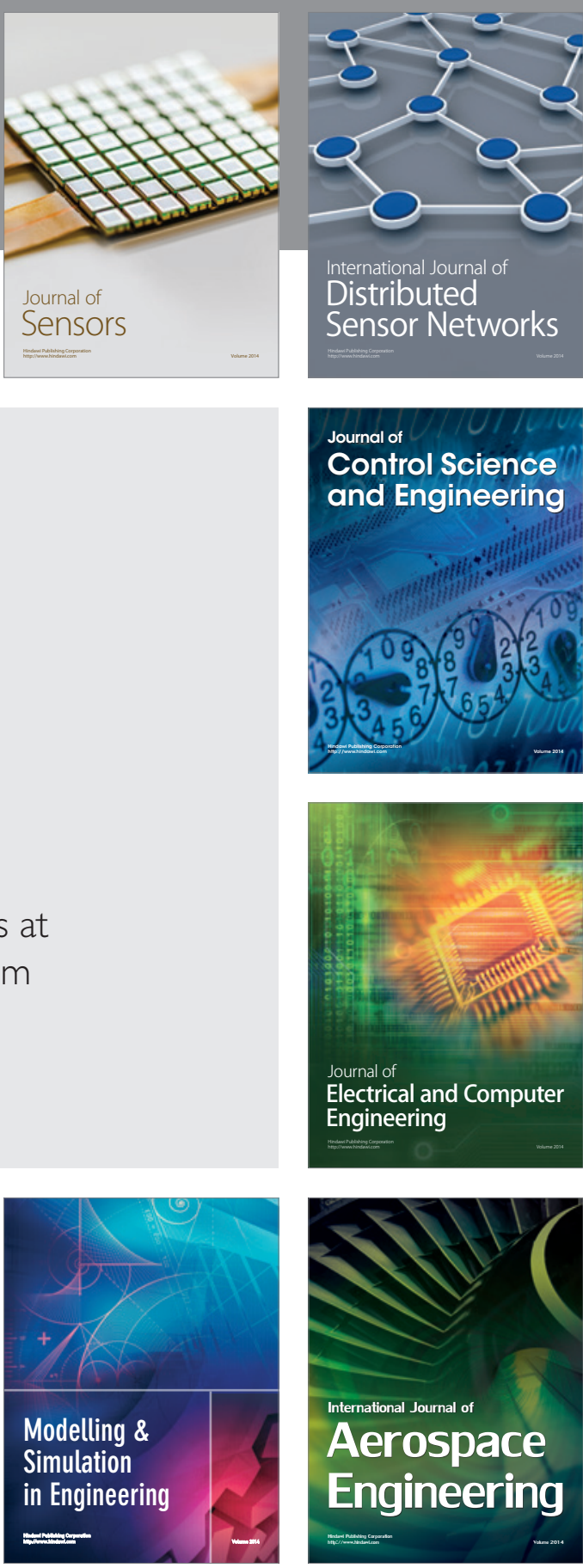

International Journal of

Distributed

Sensor Networks

$-$

Joumal of

Control Science

and Engineering
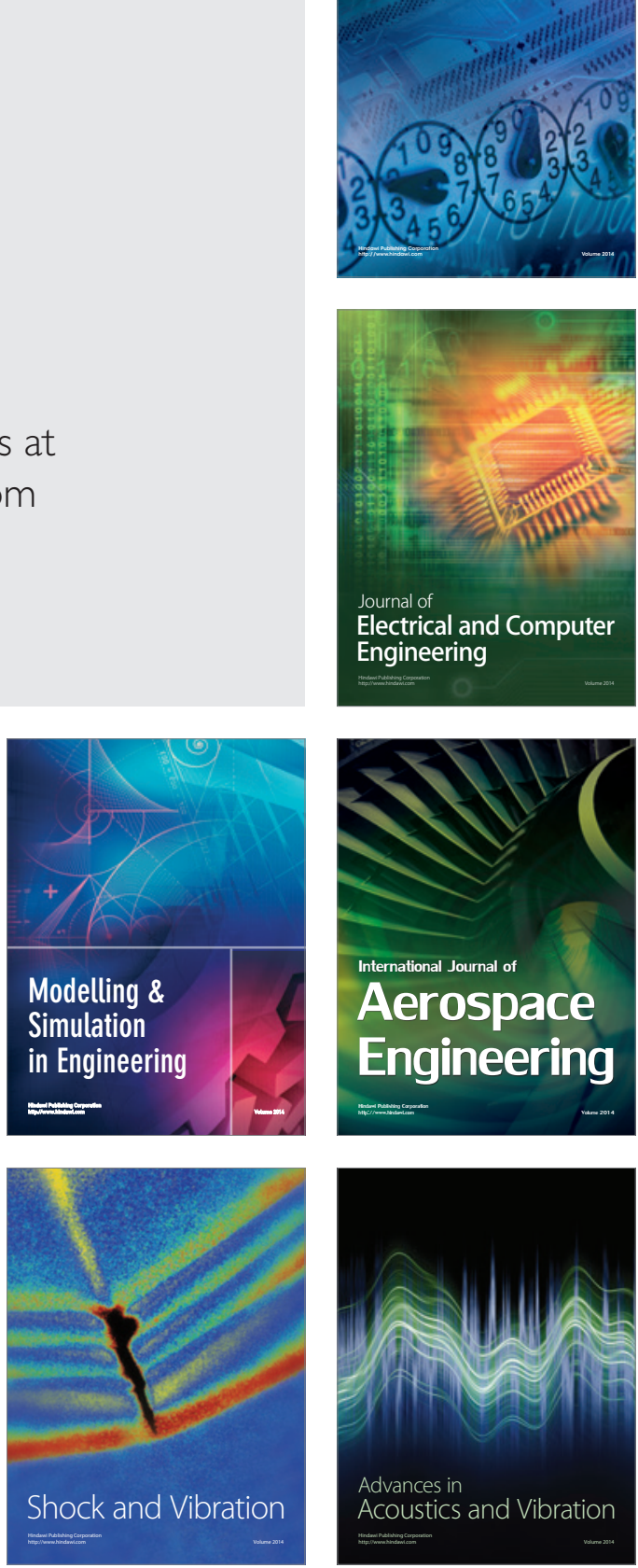